\title{
Die neue EBR-Richtlinie 2009/38/EG
}

\section{Einleitung}

Vor wenigen Monaten ist die neue Richtlinie über Europäische Betriebsräte, die Richtlinie 2009/38/EG, ${ }^{1}$ in Kraft getreten. ${ }^{2}$ Diese ändert die bestehende Richtlinie über Europäische Betriebsräte, die Richtlinie 94/45/EG, ${ }^{3}$ an einigen Punkten inhaltlich ab. ${ }^{4}$ Weitreichende Wunschvorstellungen wie sie die Gewerkschaften mit einer Revision der Richtlinie verbunden haben mögen, hat der Europäische Gesetzgeber nicht erfüllt: Weder wurden die Schwellenwerte zur Errichtung von Europäischen Betriebsräten abgesenkt, noch wurde die dreijährige „Wartefrist" für die Errichtung eines Europäischen Betriebsrats kraft Gesetzes verkürzt, noch sieht die neue Richtlinie explizite abschreckenden Sanktionen (Unterlassungsanspruch, einstweilige Verfügung) für den Fall vor, dass ein Unternehmen seinen sich aus der Richtlinie ergebenden Verpflichtungen nicht nachkommt. Dennoch wird die neue Richtlinie von Seiten der deutschen Gewerkschaften - so scheint es jedenfalls aus der Sicht eines außen stehenden Beobachters - überwiegend positiv bewertet. Die Hans Böckler Stiftung verzeichnet z. B. ,deutliche Fortschritte“. ${ }^{5}$ Zuweilen hört man auch Sätze wie „Mehr war nicht drin“6 thusiastisch klingt, aber auch nicht negativ.

In diesem Beitrag sollen einige Neuerungen vorgestellt und näher beleuchtet werden. ${ }^{7}$

\section{Geltungsbereich und Begriffsbestimmungen}

\section{Abschichtung der Leitungs- und Vertretungsebenen}

Was zunächst den Geltungsbereich der Richtlinie betrifft, so ist in Art. 1 Abs. 3 der Richtlinie nunmehr ausdrücklich davon die Rede, dass ,,die Unterrichtung und Anhörung der Arbeitnehmer ... auf der je nach behandeltem Thema relevanten Leitungs- und

1 Richtlinie 2009/38/EG des Europäischen Parlaments und des Rates vom 6. Mai 2009 über die Einsetzung eines Europäischen Betriebsrats oder die Schaffung eines Verfahrens zur Unterrichtung und Anhörung der Arbeitnehmer in gemeinschaftsweit operierenden Unternehmen und Unternehmensgruppen, Abl. L 122/28 v. 16.5.2009.

2 Zur Praxis des Wirkens Europäischer Betriebsräte zuletzt Eurofound, European Works Councils in practice: Key research findings - Background paper, 2008; im Internet: http://www.eurofound.europa.eu/publications/htmlfiles/ef0828.htm.

3 Richtlinie 94/45/EG des Rates vom 22. September 1994 über die Einsetzung eines Europäischen Betriebsrats oder die Schaffung eines Verfahrens zur Unterrichtung und Anhörung der Arbeitnehmer in gemeinschaftsweit operierenden Unternehmen und Unternehmensgruppen.

4 Zur gesetzgeberischen „Vorgeschichte“ http://www.euro-betriebsrat.de/ebr/113.php.

5 http://www.boeckler.de/29548 94705.html.

6 Helmer, Mitbestimmung 3/2009, 36.

7 Vgl. hierzu auch Melot de Beauregard/Buchmann, BB 2009, 1417. 
Vertretungsebene (erfolgt)“ ${ }^{8} \mathrm{Zu}$ diesem Zweck, so heißt es in Art. 1 Abs. 3 weiter, beschränken sich die Zuständigkeiten des Europäischen Betriebsrats und der Geltungsbereich des Verfahrens zur Unterrichtung und Anhörung der Arbeitnehmer gemäß dieser Richtlinie auf länderübergreifende Angelegenheiten. Diese wiederum werden in Art. 1 Abs. 4 verstanden als Angelegenheiten, ,die das gemeinschaftsweit operierende Unternehmen oder die gemeinschaftsweit operierende Unternehmensgruppe insgesamt oder mindestens zwei der Betriebe oder der zur Unternehmensgruppe gehörenden Unternehmen in zwei verschiedenen Mitgliedstaaten betreffen. Unter deutschen Rechtswissenschaftlern ist Art. 1 Abs. 3 als Ausdruck des Subsidiaritätsgedankens verstanden ${ }^{9}$ und der europäische Gesetzgeber für seine „Selbstbeschränkung“ gelobt worden. ${ }^{10}$

\section{Definition der „länderübergreifenden Angelegenheit“}

Was die Definition der ,länderübergreifenden Angelegenheit“ betrifft, so hat sich diese gegenüber Anhang 1 a) der alten Rechtlinie nicht verändert. Doch enthält die neue Richtlinie gewisse Anhaltspunkte für ein extensiveres Verständnis des Begriffs. In diese Richtung weist insbesondere Erwägungsgrund 12, wonach ,geeignete Vorkehrungen“ zu treffen (sind), damit die Arbeitnehmer gemeinschaftsweit operierender Unternehmen oder Unternehmensgruppen angemessen informiert und angehört werden, wenn Entscheidungen, die sich auf sie auswirken, außerhalb des Mitgliedstaats getroffen werden, in dem sie beschäftigt sind. Dies spricht dafür, eine ,länderübergreifende Angelegenheit" z. B. nicht nur dann anzunehmen, wenn eine in Spanien ansässige zentrale Leitung zum Unternehmen gehörende Betriebe in Ungarn und den Niederlanden schließt, sondern eine derartige Angelegenheit bereits dann zu bejahen, wenn nur die Schließung eines Betriebs in Ungarn im Raum steht. ${ }^{11}$

\section{Nähere Definitionen von „Unterrichtung“ und „Anhörung“}

Art. 2 Abs. 1 RL 2009/38/EG enthält nunmehr nähere Definitionen dessen, was unter „Unterrichtung“ und „Anhörung“ i. S. d. Richtlinie zu verstehen ist. „Unterrichtung“ wird definiert als „Übermittlung von Informationen durch den Arbeitgeber an die Arbeitnehmervertreter, um ihnen Gelegenheit zur Kenntnisnahme und Prüfung der behandelten Frage zu geben; die Unterrichtung erfolgt zu einem Zeitpunkt, in einer Weise und in einer inhaltlichen Ausgestaltung, die dem Zweck angemessen sind und es den Arbeitnehmervertretern ermöglichen, die möglichen Auswirkungen eingehend zu bewerten und gegebenenfalls Anhörungen mit dem zuständigen Organ des gemeinschaftsweit operierenden Unternehmens oder der gemeinschaftsweit operierenden Unternehmensgruppe vorzubereiten“ (Art. 2 Abs. 1 lit f). „Anhörung“ wird definiert als „die Ein-

8 Vgl. hierzu Melot de Beauregard/Buchmann, BB 2009, 1417 (1419) mit dem Hinweis, dass „nach bisheriger Vorstellung die EBR-Richtlinie die Regelungen des BetrVG vollständig unberührt" ließ.

9 Melot de Beauregard/Buchmann, BB 2009, 1417 (1418).

10 Thüsing, NZA 2009, 408 (411).

11 Blanke, AuR 2009, 242 (249); anders Melot de Beauregard/Buchmann, BB 2009, 1417 (1419). 
richtung eines Dialogs und den Meinungsaustausch zwischen den Arbeitnehmervertretern und der zentralen Leitung oder einer anderen, angemesseneren Leitungsebene zu einem Zeitpunkt, in einer Weise und in einer inhaltlichen Ausgestaltung, die es den Arbeitnehmervertretern auf der Grundlage der erhaltenen Informationen ermöglichen, unbeschadet der Zuständigkeiten der Unternehmensleitung innerhalb einer angemessenen Frist zu den vorgeschlagenen Maßnahmen, die Gegenstand der Anhörung sind, eine Stellungnahme abzugeben, die innerhalb des gemeinschaftsweit operierenden Unternehmens oder der gemeinschaftsweit operierenden Unternehmensgruppe berücksichtigt werden kann“" (Art. 2 Abs. 1 lit. g). ${ }^{12}$

Ersichtlich wollte der Gemeinschaftsgesetzgeber mit der Neufassung der Begriffsbestimmungen die Rechtzeitigkeit der Beteiligung sicherstellen. Der Europäische Betriebsrat soll in die Lage versetzt werden, Einfluss auf die Entscheidung der Unternehmensleitung zu nehmen. ${ }^{13}$ So deutlich diese Zielsetzung ist, so problematisch sind die vielen Unsicherheiten, die mit den neuen Begriffsbestimmungen einhergehen. Dies gilt insbesondere mit Blick auf die Definition der „Anhörung“, die nach Art. 2 Abs. 1 lit. f. „unbeschadet der Zuständigkeiten der Unternehmensleitung“ erfolgen soll. Schon jetzt ist höchst zweifelhaft, wie dieser Vorbehalt zu verstehen ist. ${ }^{14}$ Dabei tragen die Erwägungsgründe, die bekanntlich bei der Auslegung einer Richtlinie zu berücksichtigen sind, ${ }^{15}$ nicht nur wenig zur Klärung bei, sondern fördern die Unsicherheiten sogar noch weiter, indem dort z. B. davon die Rede ist, dass ,,das Recht der Arbeitnehmer oder ihrer Vertreter auf rechtzeitige Unterrichtung und Anhörung ... in vollem Umfang Beachtung findet“ (Erwägungsgrund 46), aber auch davon, dass die „Anpassungsfähigkeit“ der Unternehmen nicht beeinträchtigt werden dürfe (Erwägungsgrund 14) und die gebotene Unterrichtung nicht dazu führen dürfe, ,den Entscheidungsprozess in den Unternehmen zu verlangsamen“(Erwägungsgrund 22). ${ }^{16}$ Wieder einmal ist also alles davon abhängig, wie der Europäische Gerichtshof die entsprechenden Bestimmungen interpretieren wird.

12 Nach deutschem Recht bezeichnet, Anhörung“ den „Meinungsaustausch und die Einrichtung eines Dialogs zwischen den Arbeitnehmervertretern und der zentralen Leitung oder einer anderen geeigneten Leitungsebene“ ( $§ 1$ Abs. 4 EBRG). Diese Definition weicht vom überkommenen deutschen Sprachgebrauch ab; krit. daher Däubler/Kittner/Klebe-Däubler, § 1 EBRG Rn. 7; den Begriff „Beratungsrecht“ empfiehlt Lörcher, AuR 1996, 297. Der Begriff der „Unterrichtung“" wird bislang nicht explizit definiert; vgl. allerdings § 19 S. 1 EBRG: „Soll ein Verfahren zur Unterrichtung und Anhörung der Arbeitnehmer eingeführt werden, ist schriftlich zu vereinbaren, unter welchen Voraussetzungen die Arbeitnehmervertreter das Recht haben, die ihnen übermittelten Informationen gemeinsam zu beraten und wie sie ihre Vorschläge oder Bedenken mit der zentralen Leitung oder einer anderen geeigneten Leitungsebene erörtern können. Die Unterrichtung muss sich insbesondere auf grenzübergreifende Angelegenheiten erstrecken, die erhebliche Auswirkungen auf die Interessen der Arbeitnehmer haben".

13 So auch Thüsing, NZA 2009, 408.

14 Eine Diskussion alternativer Lesarten bei Blanke, AuR 2009, 242 (247).

15 Vgl. zuletzt Generalanwalt Mazak, Schlussantr., Slg. 2007, I-8561 Rn. 51 - Palacios.

16 Immerhin ergibt sich aus Erwgr. 44, dass eine Unterscheidung vorgenommen werden sollte „Zwischen den Bereichen, in denen eine Unterrichtung obligatorisch ist, und den Bereichen, in denen der Europäische Betriebsrat auch angehört werden muss". 


\section{Verhandlungsverfahren}

Bei der Einrichtung eines Europäischen Betriebsrats setzt die Richtlinie bekanntlich primär auf ein Verfahren der Verhandlung zwischen zentraler Leitung und einem sog. besonderen Verhandlungsgremium auf Arbeitnehmerseite.

\section{Verantwortung der Leitung}

Nach Art. 4 Abs. 4 ist nunmehr die „Leitung eines zu einer gemeinschaftsweit operierenden Unternehmensgruppe gehörenden Unternehmens ... dafür verantwortlich, die für die Aufnahme von Verhandlungen ... erforderlichen Informationen zu erheben und an die Parteien, auf die die Richtlinie Anwendung findet, weiterzuleiten, insbesondere die Informationen in Bezug auf die Struktur des Unternehmens oder der Gruppe und die Belegschaft". Mit dieser Vorschrift wird der Rechtsprechung des EuGH in den Rechtssachen Bofrost, ${ }^{17}$ Kühne \& Nagel ${ }^{18}$ sowie ADS-Anker ${ }^{19}$ Rechnung getragen. ${ }^{20}$

\section{Zusammensetzung des besonderen Verhandlungsgremiums}

Mit Blick auf die Zusammensetzung des besonderen Verhandlungsgremiums sieht Art. 5 Abs. 2 lit. b nunmehr vor, dass dessen „Mitglieder entsprechend der Zahl der in jedem Mitgliedstaat beschäftigen Arbeitnehmer des gemeinschaftsweit operierenden Unternehmens oder der gemeinschaftsweit operierenden Unternehmensgruppe gewählt oder bestellt (werden), so dass pro Mitgliedstaat für jeden Anteil der in diesem Mitgliedstaat beschäftigten Arbeitnehmer, der $10 \%$ der Gesamtzahl der in allen Mitgliedstaaten beschäftigten Arbeitnehmer entspricht, oder für einen Bruchteil dieser Tranche Anspruch auf einen Sitz besteht". Dies entspricht der Bestimmung in Art. 3 Abs. 2

17 EuGH v. 29.3.2001 - Rs. C-62/99, NZA 2001, 506; näher hierzu etwa Junker, RdA 2002, 32; Ritzberger-Moser, ZESAR 2002, 85. In der Rechtssache Bofrost hatte der EuGH u. a. entschieden, dass ein Unternehmen einer Unternehmensgruppe auch dann zur Auskunftserteilung an die Organe der internen Arbeitnehmervertretung verpflichtet ist, wenn noch nicht feststeht, ob es sich bei der Unternehmensleitung, an die sich die Arbeitnehmer wenden, um die Leitung eines innerhalb der Unternehmensgruppe herrschenden Unternehmens handelt; näher zu diesem „,vorgelagerten Informationsanspruch“ Däubler/Kittner/Klebe-Däubler, § 5 EBRG Rn. 1; MünchArbR/Joost, 3. Aufl. 2009, § 274 Rn. 44; vgl. auch Thüsing/Leder, SAE 2002, 171.

18 EuGH v. 13.1.2004-Rs. C-440/00, NZA 2004, 160; vgl. hierzu BAG, BB 2005, 440 m. Anm. Leder/Zimmer. Zur Problematik der Mitwirkung der Arbeitnehmer in einem globalen Logistikunternehmen Hansen, ZfA 2005, 225.

19 EuGH v. 15.7.2004 - Rs. C-349/01, NZA 2004, 1167.

20 Näher zu den Auskunftsansprüchen der Arbeitnehmervertretung zur Errichtung eines Europäischen Betriebsrats bei Unternehmensgruppen Joost, BB 2001, 2214; zum Ganzen auch Däubler, BB 2004, 441; Krause, BB-Special 2004, 23; Coen, AuR 2002, 30, Waas, ECL 2005, 138. 
lit. a i. RL 2001/86/EG. ${ }^{21}$ Eine ursprünglich vorgesehene Bestimmung, wonach ein Sitz erst ab 50 Arbeitnehmern gewährt werden sollte, wurde im Gesetzgebungsverfahren gestrichen. Dementsprechend wurde in Deutschland bereits kritisch bemerkt, die Neuregelung führe zu einer „Aufblähung“ des besonderen Verhandlungsgremiums und zu einer „Überrepräsentation“ kleiner Belegschaften“22 mit, so wird man hinzufügen dürfen, einer höheren Kostenbelastung auf Seiten der Unternehmen. Umgekehrt wird die in der Neuregelung enthaltene Stärkung des Grundsatzes der Proportionalität von manchen als „Schritt in die richtige Richtung“ angesehen. ${ }^{23}$ Im deutschen Recht gilt - im Einklang mit Art. 5 Abs. 2 lit. v RL 94/45/EG - der sog. Grundsatz der Repräsentativität, wonach aus jedem Mitgliedstaat, in dem das Unternehmen oder die Unternehmensgruppe einen Betrieb hat, ein Arbeitnehmervertreter in das besondere Verhandlungsgremium entsandt wird ( $\$ 10$ Abs. 1 EBRG). Wenn bestimmte Quoren überschritten werden, sind nach $\S 10$ Abs. 2 EBRG zusätzliche Vertreter in das Gremium zu entsenden. ${ }^{24}$ Damit werden die Grundsätze der Repräsentativität und der Proportionalität ${ }^{25}$ miteinander verbunden. ${ }^{26}$ Zuständig für die Bestellung der inländischen Arbeitnehmervertreter ist übrigens grundsätzlich der Gesamt- bzw. der Konzernbetriebsrat ( $\$ 11$ Abs. 1 u. 2 BetrVG). Anders als nach dem für die Arbeitnehmermitbestimmung in der

21 Richtlinie 2001/86/EG des Rates vom 8. Oktober 2001 zur Ergänzung des Statuts der Europäischen Gesellschaft hinsichtlich der Beteiligung der Arbeitnehmer, ABl. Nr. L 294 S. 22. Art. 5 Abs. 2 lit. c sieht vor, dass „,die Zusammensetzung des besonderen Verhandlungsgremiums und der Beginn der Verhandlungen ... der zentralen Leitung und den örtlichen Unternehmensleitungen sowie den zuständigen europäischen Arbeitnehmer- und Arbeitgeberverbänden mitgeteilt (werden)“. §9 Abs. 3 EBRG lautet: Die zentrale Leitung hat die Antragsteller, die örtlichen Betriebs- oder Unternehmensleitungen, die dort bestehenden Arbeitnehmervertretungen sowie die in inländischen Betrieben vertretenen Gewerkschaften über die Bildung eines besonderen Verhandlungsgremiums und seine Zusammensetzung zu unterrichten“. Überdies bestimmt $\S 12$ EBRG: „Der zentralen Leitung sind unverzüglich die Namen der Mitglieder des besonderen Verhandlungsgremiums, ihre Anschriften sowie die jeweilige Betriebszugehörigkeit mitzuteilen. Die zentrale Leitung hat die örtlichen Betriebsoder Unternehmensleitungen, die dort bestehenden Arbeitnehmervertretungen sowie die in inländischen Betrieben vertretenen Gewerkschaften über diese Angaben zu unterrichten“.

22 Thüsing, NZA 2009, 408 (411).

23 Melot de Beauregard/Buchmann, BB 2009, 1417 (1420).

24 Vgl. im Übr. § 11 Abs. 5 EBRG, wonach Frauen und Männer entsprechend ihrem zahlenmäBigen Verhältnis bestellt werden.

25 Näher zu diesem Grundsatz (auch zu den insoweit verschiedentlich geäußerten europarechtlichen Bedenken) MünchArbR/Joost, 3. Aufl. 2009, § 274 Rn. 50.

26 Däubler/Kittner/Klebe-Däubler, § 10 EBRG Rn. 1 f. 
Europäischen Aktiengesellschaft (SEBG) ${ }^{27}$ geltenden Gesetz sind keine Sitze für Gewerkschaftsvertreter reserviert. ${ }^{28}$

\section{Vor- und Nachbereitung der Sitzungen}

Nach Art. 5 Abs. 4 Uabs. 2 ist das besondere Verhandlungsgremium, ,vor und nach jeder Sitzung mit der zentralen Leitung ... berechtigt, zu tagen, ohne dass Vertreter der zentralen Leitung dabei zugegen sind, und dabei die erforderlichen Kommunikationsmittel zu nutzen". In der Praxis dürften aber schon heute jedenfalls interne Nachbereitungssitzungen weithin üblich sein. ${ }^{29} \mathrm{Im}$ Übrigen bestimmt $\S 13$ Abs. 2 EBRG für das deutsche Recht, dass vor jeder Verhandlung mit der zentralen Leitung das besondere Verhandlungsgremium das Recht hat, eine Sitzung durchzuführen und zu dieser einzuladen“. Das besondere Verhandlungsgremium darf also vor jeder Verhandlung mit der zentralen Leitung eine interne Vorbereitungssitzung durchführen, wobei die Sitzungen nach $\S 8$ Abs. 3 S. 2 EBRG mit der zentralen Leitung einvernehmlich festzulegen sind. ${ }^{30}$ Darauf, dass diese Sitzungen für die interne Abstimmung und die Vorbereitung der Verhandlungen mit der zentralen Leitung große Bedeutung haben, wird in der Lit. immer wieder hingewiesen. ${ }^{31}$

\section{Rolle der europäischen Gewerkschaften}

Eine Anerkennung der Rolle der europäischen Gewerkschaften findet sich in Art. 5 Abs. 4 Uabs. 3 RL 2009/38/EG. ${ }^{32}$ Danach kann das besondere Verhandlungsgremium bei den Verhandlungen Sachverständige seiner Wahl hinzuziehen, zu denen Vertreter der kompetenten anerkannten Gewerkschaftsorganisationen auf Gemeinschaftsebene gehören können, um sich von ihnen bei seiner Arbeit unterstützen zu lassen. Diese Sachverständigen und Gewerkschaftsvertreter können auf Wunsch des besonderen Verhandlungsgremiums den Verhandlungen in beratender Funktion beiwohnen. Nach deut-

27 Nach $\S 6$ Abs. 3 SEBG ist jedes dritte Mitglied ein Vertreter einer Gewerkschaft, die in einem an der Gründung der SE beteiligten Unternehmen vertreten ist, wenn dem besonderen Verhandlungsgremium mehr als zwei Mitglieder aus dem Inland angehören.

28 Dabei sollte es nach Auffassung mancher auch bleiben; so dezidiert Thüsing, NZA 2009, 408 (412) u. Hinweis auf europarechtliche Bedenken gegen § 6 Abs. 3 SEBG; näher etwa Lunk/ Hinrichs, NZA 2007, 773 (777), wonach die SE-Richtlinie zwar den Mitgliedstaaten Spielraum für die Anordnung der Wahl von unternehmensexternen Gewerkschaftsvertretern in das besondere Verhandlungsgremium eröffne, jedoch keine Ermächtigung zur Regelung einer zwingenden Vertretung der Arbeitnehmer durch - unternehmensexterne - Gewerkschaftsvertreter enthalte.

29 So Blanke, AuR 2009, 242 (248).

30 Vgl. MünchArbR/Joost, 3. Aufl. 2009, § 274 Rn. 71; auch Däubler/Kittner/Klebe-Däubler, $\S 13$ EBRG Rn. 7, der insoweit unter Hinweis auf die ,eigenverantwortliche Wahrnehmung der Aufgaben“ und die „Rolle als gleichwertiger Verhandlungspartner“ annimmt, dass Vorschläge des besonderen Verhandlungsgremiums von der zentralen Leitung nur abgelehnt werden können, wenn „nachvollziehbare erhebliche Gründe“ vorliegen.

31 Vgl. nur Däubler/Kittner/Klebe-Däubler, § 13 EBRG Rn. 7.

32 Kritisch insoweit Melot de Beauregard/Buchmann, BB 2009, 1417 (1421). 
schem Recht gilt - ebenso wie in vielen anderen Rechtsordnungen ${ }^{33}$ - schon heute, dass sich das besondere Verhandlungsgremium durch Sachverständige seiner Wahl unterstützen lassen kann, soweit dies zur ordnungsgemäßen Erfüllung seiner Aufgaben erforderlich ist (§ 13 Abs. 4 S. 1 EBRG). Sachverständige können dabei ausdrücklich auch Beauftragte von Gewerkschaften sein (§ 13 Abs. 4 S. 2 EBRG) ${ }^{34}$ wobei - anders als nach deutschem Betriebsverfassungsrecht ( $\$ 80$ Abs. 3 BetrVG) - eine nähere Vereinbarung mit dem Arbeitgeber insoweit nicht erforderlich ist. ${ }^{35}$ Insoweit ist mit Blick auf das alte Recht im Schrifttum kritisch angemerkt worden, dass das deutsche Recht hinter den Anforderungen der Richtlinie zurückbleibe, da diese (in Art. 5 Abs. 4 S. 3) die Einschaltung von Sachverständigen an keine besonderen Voraussetzungen knüpfe. Dementsprechend wird die Auffassung vertreten, $§ 13$ Abs. 4 S. 1 EBRG müsse in richtlinienkonformer Auslegung so verstanden werden, dass die Erforderlichkeit der Hinzuziehung von Sachverständigen o. W. angenommen werden könne und nicht weiter begründet werden müsse. Die Einschaltung von Sachverständigen sei bei Verhandlungen wegen der Schwierigkeit der Materie immer zur ordnungsgemäßen Aufgabenerfüllung erforderlich. ${ }^{36}$

\section{Inhalt der Vereinbarungen}

Hinsichtlich des Inhalts der zwischen zentraler Leitung und besonderem Verhandlungsgremium abzuschließenden Vereinbarung, über die nach Art. 6 Abs. 1 ,im Geiste der Zusammenarbeit“" zu verhandeln ist, ${ }^{37}$ bekräftigt Art. 6 Abs. 2 zunächst die „Autonomie der Parteien“" 38 um dann doch einige inhaltliche Vorgaben zu machen.

\section{Sicherstellung einer ,ausgewogenen Vertretung“}

Art. 6 Abs. 2 lit. b fordert mit Blick auf den Inhalt der zwischen zentraler Leitung und besonderem Verhandlungsgremium zu schließenden Vereinbarung auch Regelungen, die „so weit als möglich eine ausgewogene Vertretung der Arbeitnehmer nach Tätigkeit, Arbeitnehmerkategorien und Geschlecht" sicherstellen. Mit dieser Regelung macht der

33 EBRG-Kommentar/Blanke, § 14 Rn. 23.

34 Vgl. in diesem Zusammenhang auch § 16 Abs. 1 EBRG: „Die durch die Bildung und Tätigkeit des besonderen Verhandlungsgremiums entstehenden Kosten trägt die zentrale Leitung. Werden Sachverständige nach $\S 13$ Abs. 4 hinzugezogen, beschränkt sich die Kostentragungspflicht auf einen Sachverständigen. Die zentrale Leitung hat für die Sitzungen in erforderlichem Umfang Räume, sachliche Mittel, Dolmetscher und Büropersonal zur Verfügung zu stellen sowie die erforderlichen Reise- und Aufenthaltskosten der Mitglieder des besonderen Verhandlungsgremiums zu tragen“".

35 Vgl. hierzu nur MünchArbR/Joost, 3. Aufl. 2009, § 275 Rn. 30.

36 So Däubler/Kittner/Klebe-Däubler, § 13 EBRG Rn. 11; a. A. MünchArbR/Joost, 3. Aufl. 2009, § 274 Rn. 74.

37 Vgl. auch § 8 Abs. 3 S. 1 EBRG: „Die zentrale Leitung und das besondere Verhandlungsgremium arbeiten vertrauensvoll zusammen".

38 Vgl. § 17 S. 1 Hs. 1 EBRG: „Die zentrale Leitung und das besondere Verhandlungsgremium können frei vereinbaren, wie die grenzübergreifende Unterrichtung und Anhörung der Arbeitnehmer ausgestaltet wird“". 
Gemeinschaftsgesetzgeber klar, dass die Beteiligten an die entsprechenden Diskriminierungsverbote gebunden sind. Doch dürfte dies die Rechtsfertigungsgründe mit einschließen, so dass die Beteiligten aus sachlichen Gründen Abweichendes vereinbaren können. ${ }^{39}$

\section{Abgrenzung der Kompetenzen der Beteiligungsorgane}

Mit Blick auf die erforderliche Abgrenzung der Kompetenzen der Beteiligungsorgane sieht Art. 6 Abs. 2 lit. c vor, dass die Vereinbarung auch Regelungen über die Zuständigkeiten von Europäischem Betriebsrat auf der einen und den einzelstaatlichen Arbeitnehmervertretungen auf der anderen Seite enthalten soll. ${ }^{40}$ Allerdings können die Beteiligten dabei nicht von dem in Art. 1 Abs. 3 der Richtlinie aufgestellten Grundsatz abweichen, wonach sich die Zuständigkeiten des Europäischen Betriebsrats auf „länderübergreifende Angelegenheiten“" beschränken.

\section{Sog. engerer Ausschuss}

Art. 6 Abs. 2 lit. e nennt als weiteren Gegenstand einer Regelung in der Vereinbarung - dem Vorbild in Anhang Auffangregelung Teil I lit. c RL 2001/86/EG folgend - Bestimmungen über einen innerhalb des Europäischen Betriebsrats einzusetzenden sog. engeren Ausschuss“. ${ }^{41}$ Das deutsche Recht sieht insoweit vor, dass der Europäische Betriebsrat aus seiner Mitte einen Ausschuss von drei Mitgliedern bildet, wenn ihm neun oder mehr Mitglieder angehören. Dieser Ausschuss führt die laufenden Geschäfte des Europäischen Betriebsrats ( 26 Abs. 1 EBRG). Laufende Geschäfte in diesem Sinne sind v. a. die Vorbereitung der Treffen mit der zentralen Leitung sowie der Vorbesprechungen und der nachbereitenden Sitzungen des Europäischen Betriebsrats. ${ }^{42}$ Außerdem wirkt der Ausschuss bei der Anhörung und Unterrichtung über ,,außergewöhnliche Umstände“ mit, sofern diese „erhebliche Auswirkungen auf die Interessen der Arbeitnehmer haben“" (§ 33 Abs. 1 u. 2 S. 1 EBRG). ${ }^{43}$ Dem Ausschuss gehören, neben dem Vorsitzenden, zwei weitere zu wählende Mitglieder an, wobei die Mitglieder des Ausschusses in verschiedenen Mitgliedstaaten beschäftigt sein sollen (§26 Abs. 2 EBRG).

39 Ebenso Thüsing, NZA 2009, 408 (410).

40 Vgl. insoweit auch Art. 12 der Richtlinie.

41 Vgl. Erwgr. 30: „Vereinbarungen müssen gegebenenfalls die Einsetzung und die Arbeit eines engeren Ausschusses vorsehen, damit eine Koordinierung und eine höhere Effizienz der regelmäßigen Arbeit des Europäischen Betriebsrats sowie eine schnellstmögliche Unterrichtung und Anhörung im Falle außergewöhnlicher Umstände ermöglicht wird“. Erwgr. 44 bestimmt überdies: „Damit der engere Ausschuss die erforderliche Koordinierungsrolle wahrnehmen und im Falle außergewöhnlicher Umstände effizient handeln kann, muss dieser Ausschuss bis zu fünf Mitglieder umfassen und regelmäßig beraten können“".

42 Däubler/Kittner/Klebe-Däubler, § 26 EBRG Rn. 3; vgl. auch MünchArbR/Joost, 3. Aufl. $2009, \S 275$ Rn. 20.

43 Däubler/Kittner/Klebe-Däubler, § 33 EBRG Rn. 7; MünchArbR/Joost, 3. Aufl. 2009, § 275 Rn. 62. 


\section{Strukturelle Änderungen}

Art. 13 sieht (umfassende) ${ }^{44}$ Neuverhandlungen und ggf. eine Anpassung der bestehenden Vereinbarung für den Fall vor, dass ,sich die Struktur des gemeinschaftsweit operierenden Unternehmens oder der gemeinschaftsweit operierenden Unternehmensgruppe wesentlich (ändert)“. Eine Definition des Begriffs ,strukturelle Änderung“ fehlt in Art. 13. Doch nennt Erwägungsgrund 40 beispielhaft die Fälle von Fusion, Übernahme und Spaltung. Auch der Erwerb einer Mehrheitsbeteiligung soll nach mancher Ansicht eine ,strukturelle Änderung“ in diesem Sinne sein. ${ }^{45}$ Dieser Auffassung war jedenfalls der zuständige Berichterstatter im Europäischen Parlament, der Abgeordnete BushillMatthews. ${ }^{46}$

In diesem Zusammenhang mag auch von Interesse sein, dass nach der zum 19. 8. 2008 in Kraft getretenen Nr. 9 a des $\S 106$ BetrVG, zu den wirtschaftlichen Angelegenheiten, über die der Wirtschaftsausschuss nach $\S 106$ Abs. 2 BetrVG zu unterrichten ist, auch die Übernahme des Unternehmens zählt, wenn hiermit der Erwerb der Kontrolle verbunden ist. Außerdem wird mit $\S 109$ a BetrVG subsidiär die Unterrichtung des Betriebsrats vorgesehen, soweit kein Wirtschaftsausschuss besteht. Zur Konkretisierung verweist die Gesetzesbegründung auf $\S 29$ Abs. 2 WpÜG, wonach eine Kontrolle des Unternehmens insbesondere vorliegt, wenn mindestens $30 \%$ der Stimmrechte an dem Unternehmen gehalten werden. ${ }^{47}$ Die Regelung passt allerdings nur für börsennotierte Unternehmen.

\section{Sonstige Bestimmungen}

\section{Kosten und Sachaufwand}

Nach Art. 10 Abs. 1 RL 2009/38/EG gilt nunmehr, dass, „unbeschadet der Zuständigkeiten der anderen Gremien oder Organisationen in diesem Bereich“, die Mitglieder des Europäischen Betriebsrats ,über die Mittel (verfügen), die erforderlich sind, um die Rechte auszuüben, die sich aus dieser Richtlinie ergeben, um kollektiv die Interessen der Arbeitnehmer des gemeinschaftsweit operierenden Unternehmens oder der gemeinschaftsweit operierenden Unternehmensgruppe zu vertreten“. Nach deutschem Recht gilt aber ohnehin, dass die durch die Bildung und Tätigkeit des Europäischen Betriebsrats entstehenden Kosten die zentrale Leitung trägt (§30 S. 1 EBRG). Die zentrale Lei-

44 Kritisch insoweit CEP v. 29.8.2008, S. 4.

45 Vgl. insoweit Hall/Carley, European Works Councils and transnational restructuring, 2007; im Internet: http://www.eurofound.europa.eu/publications/htmlfiles/ef0683.htm.

46 Bericht über den Vorschlag für eine Richtlinie des Europäischen Parlaments und des Rates über die Einsetzung eines Europäischen Betriebsrats oder die Schaffung eines Verfahrens zur Unterrichtung und Anhörung der Arbeitnehmer in gemeinschaftsweit operierenden Unternehmen und Unternehmensgruppen (Neufassung) (KOM [2008] 0419 - C6-0258/2008 2008/0141[COD]), A6-0454/2008, S. 8; a. A. Thüsing, NZA 2009, 408 (411).

47 BT-Drs. 16/7438, S. 15. 
tung hat insbesondere für die Sitzungen ${ }^{48}$ und die laufende Geschäftsführung in erforderlichem Umfang Räume, sachliche Mittel und Büropersonal, für die Sitzungen außerdem Dolmetscher zur Verfügung zu stellen. Auch trägt sie die erforderlichen Reiseund Aufenthaltskosten der Mitglieder des Europäischen Betriebsrats (§30 S. 3 u. 4 EBRG). Was die Hinzuziehung von Sachverständigen betrifft, so sieht $§ 29$ S. 1 EBRG vor, dass sich der Europäische Betriebsrat durch Sachverständige seiner Wahl unterstützen lassen kann, soweit dies zur ordnungsgemäßen Erfüllung seiner Aufgaben erforderlich ist. Sachverständige können dabei auch Beauftragte von Gewerkschaften sein (§ 29 S. 2 EBRG). Die Kostentragungspflicht auf Arbeitgeberseite ist allerdings auf einen Sachverständigen beschränkt ( $\$ 30$ S. 2 EBRG). Was die in Art. 10 Abs. 1 RL 2009/38/EG enthaltene Regelung anbelangt, ist zweifelhaft, ob und inwieweit hiervon auch Kosten der Rechtsdurchsetzung in gerichtlichen Verfahren erfasst werden. ${ }^{49}$ Nach deutschem Betriebsverfassungsrecht gilt jedenfalls, dass der Arbeitgeber nach $\S 40$ Abs. 1 BetrVG im erforderlichen Umfang auch die Kosten für die Rechtsdurchsetzung des Betriebsrats zu übernehmen hat. ${ }^{50}$ Da im Beschlussverfahren Gerichtskosten nicht anfallen ( $§ 12$ Abs. 5 ArbGG), geht es insoweit allerdings nur um die außergerichtlichen Kosten.

\section{Umfassende Kooperationspflicht}

Art. 10 Abs. 2 RL 2009/38/EG legt den Mitgliedern des Europäischen Betriebsrats eine umfassende Verpflichtung zur Kooperation mit den „nationalen Arbeitnehmervertretern“ auf. Danach „informieren die Mitglieder des Europäischen Betriebsrats die Arbeitnehmervertreter der Betriebe oder der zur gemeinschaftsweit operierenden Unternehmensgruppe gehörenden Unternehmen oder, in Ermangelung solcher Vertreter, die Belegschaft insgesamt ${ }^{51}$ über Inhalt und Ergebnisse der gemäß dieser Richtlinie durchgeführten Unterrichtung und Anhörung“. §35 Abs. 1 EBRG sieht insoweit bereits vor, dass der Europäische Betriebsrat den örtlichen Arbeitnehmervertretern oder, wenn es diese nicht gibt, den Arbeitnehmern der Betriebe oder Unternehmen über die Unterrichtung und Anhörung Bericht zu erstatten hat.

\section{Schutz der Arbeitnehmervertreter}

Nach Art. 10 Abs. 3 Uabs. 1 RL 2009/38/EG genießen die Mitglieder des besonderen Verhandlungsgremiums, die Mitglieder des Europäischen Betriebsrats und die Arbeitnehmervertreter, die bei dem Unterrichtungs- und Anhörungsverfahren nach Artikel 6

48 Vgl. § 27 Abs. 1 S. 1 - 3 EBRG: Der Europäische Betriebsrat hat das Recht, im Zusammenhang mit der Unterrichtung durch die zentrale Leitung nach § 32 eine Sitzung durchzuführen und zu dieser einzuladen. Das gleiche gilt bei einer Unterrichtung über außergewöhnliche Umstände nach § 33. Der Zeitpunkt und der Ort der Sitzungen sind mit der zentralen Leitung abzustimmen. Mit Einverständnis der zentralen Leitung kann der Europäische Betriebsrat weitere Sitzungen durchführen.

49 Zurückhaltend Blanke, AuR 2009, 242 (249).

50 Vgl. nur Richardi/Thüsing § 40 BetrVG Rn. 16.

51 Vgl. insoweit auch Erwgr. 33. 
Abs. 3 mitwirken, bei der Wahrnehmung ihrer Aufgaben den gleichen Schutz und gleichartige Sicherheiten wie die Arbeitnehmervertreter nach den innerstaatlichen Rechtsvorschriften und/oder Gepflogenheiten des Landes, in dem sie beschäftigt sind.

In Umsetzung dieser Regelung sieht $\S 40$ Abs. 1 EBRG vor, dass für die Mitglieder eines Europäischen Betriebsrats, die im Inland beschäftigt sind, $\S 37$ Abs. 1 bis 5 und $\S \S 78$ und 103 des Betriebsverfassungsgesetzes sowie $\S 15$ Abs. 1 und 3 bis 5 des Kündigungsschutzgesetzes entsprechend gelten. Nach § 40 Abs. 2 EBRG gilt Abs. 1 überdies entsprechend für die Mitglieder des besonderen Verhandlungsgremiums und die Arbeitnehmervertreter im Rahmen eines Verfahrens zur Unterrichtung und Anhörung. Im Einzelnen bedeutet dies, dass die genannten Arbeitnehmervertreter, ebenso wie die Mitglieder eines Betriebsrats, ihr Amt unentgeltlich als Ehrenamt führen (§ 37 Abs. 1 BetrVG). Teilt man die Bedenken, die von manchen Autoren in Deutschland zuletzt in rechtspolitischer Hinsicht gegen das Ehrenamtsprinzip geltend gemacht wurden, ${ }^{52}$ würde man diese allerdings auch (und sogar erst recht) auf den Bereich des Wirkens Europäischer Betriebsräte erstrecken müssen. Nach $\S 37$ Abs. 2 BetrVG sind die Mitglieder des Betriebsrats von ihrer beruflichen Tätigkeit ohne Minderung des Arbeitsentgelts zu befreien, wenn und soweit es nach Umfang und Art des Betriebs zur ordnungsgemäßen Durchführung ihrer Aufgaben erforderlich ist. Das gilt auch für die Mitglieder eines Europäischen Betriebsrats. Überdies hat das Mitglied eines Europäischen Betriebsrats zum Ausgleich für Betriebsratstätigkeit, die aus betriebsbedingten Gründen außerhalb der Arbeitszeit durchzuführen ist, Anspruch auf entsprechende Arbeitsbefreiung unter Fortzahlung des Arbeitsentgelts ( $\$ 37$ Abs. 3 BetrVG). Dabei darf das einschließlich eines Zeitraums von einem Jahr nach Beendigung der Amtszeit nicht geringer bemessen werden als das Arbeitsentgelt vergleichbarer Arbeitnehmer mit betriebsüblicher beruflicher Entwicklung ( $\$ 37$ Abs. 4 S. 1 BetrVG). Schließlich dürfen auch Mitglieder eines Europäischen Betriebsrats, vorbehaltlich zwingender betrieblicher Notwendigkeiten, einschließlich eines Zeitraums von einem Jahr nach Beendigung der Amtszeit nur mit Tätigkeiten beschäftigt werden, die den Tätigkeiten der in Absatz 4 genannten Arbeitnehmer gleichwertig sind ( 37 Abs. 5 BetrVG). Darüber hinaus genießen die Mitglieder eines Europäischen Betriebsrats denselben Schutz wie ihre Kollegen im Betriebsrat: Nach $\S 78$ S. 1 BetrVG dürfen die Mitglieder des Betriebsrats in der Ausübung ihrer Tätigkeit nicht gestört oder behindert werden. Nach § 78 S. 2 BetrVG dürfen sie überdies wegen ihrer Tätigkeit nicht benachteiligt oder begünstigt werden; dies gilt auch für ihre berufliche Entwicklung. Ihren Kollegen im Betriebsrat gleich stehen die Mitglieder des Europäischen Betriebsrats auch in kündigungsschutzrechtlicher Hinsicht. Nach $\S 103$ Abs. 1 BetrVG bedarf die außerordentliche Kündigung von Mitgliedern des Betriebsrats der Zustimmung des Betriebsrats. Verweigert der Betriebsrat seine Zustimmung, so kann das Arbeitsgericht sie auf Antrag des Arbeitgebers ersetzen, wenn die außerordentliche Kündigung unter Berücksichtigung aller Umstände gerechtfertigt ist ( $\$ 103$ Abs. 2 S. 1 BetrVG).

52 Rieble, NZA 2008, 276; a. A. Fischer, NZA 2007, 484. 


\section{Schulungsveranstaltungen}

Art. 10 Abs. 4 RL 2009/38/EG sieht darüber hinaus nunmehr vor, dass die Mitglieder des Europäischen Betriebsrats (und die Mitglieder des besonderen Verhandlungsgremiums), ,in dem Maße, wie dies zur Wahrnehmung ihrer Vertretungsaufgaben in einem internationalen Umfeld erforderlich ist", ... Schulungen erhalten, ohne dabei Lohnbzw. Gehaltseinbußen zu erleiden“. Einen derartigen Anspruch kannte die Richtlinie in ihrer alten Fassung nicht; diese Neuregelung zählt daher in der Tat zu den ,greifbarsten“ Änderungen der neuen Richtlinie. ${ }^{53}$ Dementsprechend war in der in $\S 40$ EBRG enthaltenen Verweisung auf $\S 37$ BetrVG bislang Abs. 6 dieser Bestimmung ausgenommen, demzufolge jedes Mitglied des Betriebsrats während seiner regelmäßigen Amtszeit Anspruch auf bezahlte Freistellung für insgesamt drei Wochen zur Teilnahme an Schulungs- und Bildungsveranstaltungen hat, die von der zuständigen obersten Arbeitsbehörde des Landes nach Beratung mit den Spitzenorganisationen der Gewerkschaften und der Arbeitgeberverbände als geeignet anerkannt sind (§ $37 \mathrm{~S} .1$ BetrVG). In der Lit. wurde dennoch schon zum alten Recht verschiedentlich vom Bestehen eines Anspruchs auf den Besuch von Schulungs- und Bildungsveranstaltungen ausgegangen. Gestützt wurde ein derartiger Anspruch auf $\S 37$ Abs. 2 BetrVG (in richtlinienkonformer Auslegung) i. V. m. $§ 40$ Abs. 1 EBRG. ${ }^{54}$

\section{Schluss}

Der europäische Gesetzgeber hat die bestehende Richtlinie über Europäische Betriebsräte nur punktuell geändert, wobei er in dem Sinne eine gewisse Vereinheitlichung erreicht hat, als die einzelnen Regelungen an die entsprechenden Vorschriften der Richtlinie 2001/86/EG angenähert wurden. Die moderaten Änderungen der Richtlinie lösen in Deutschland nur einen überschaubaren Umsetzungsbedarf aus. Dieser betrifft vornehmlich die Vorschriften über die Zusammensetzung des besonderen Verhandlungsgremiums, das Recht zur Teilnahme an Fortbildungsmaßnahmen und die Pflicht zu Neuverhandlungen bei Strukturänderungen. Manche Änderung - man denke etwa an das Recht auf die Hinzuziehung von Sachverständigen und die weitgehende Kostentragungspflicht des Arbeitgebers - hat das deutsche Recht gewissermaßen ,schon vorweggenommen“. Im Übrigen ist aber zu beachten, dass in deutschen Unternehmen und Unternehmensgruppen eine Vielzahl von Vereinbarungen nach Art. 13 Abs. 1 RL 94/45/EG abgeschlossen wurden. Diese Vereinbarungen nimmt Art. 14 Abs. 1 lit a der neuen Richtlinie aber - vorbehaltlich der Anpassungspflicht bei Strukturänderungen - vom Geltungsbereich der Richtlinie gerade aus. Auch aus diesem Grund dürften die Auswirkungen des neuen Rechts begrenzt bleiben.

53 So Melot de Beauregard/Buchmann, BB 2009, 1417 (1421).

54 Däubler/Kittner/Klebe-Däubler, § 40 EBRG Rn. 2. 